\title{
Effect of an Educational Intervention About Dietary Approach to Stop Hypertension (DASH) on Changing Knowledge, Attitude and Blood Pressure of Hypertensive Patients Attending Zagazig University Hospitals
}

\author{
Eman E. Orabi \\ Public Health and Community Medicine Department, Faculty of Medicine, Zagazig University, Zagazig City, Egypt
}

Email address:

dr.eman.elshahat@gmail.com

To cite this article:

Eman E. Orabi. Effect of an Educational Intervention About Dietary Approach to Stop Hypertension (DASH) on Changing Knowledge, Attitude and Blood Pressure of Hypertensive Patients Attending Zagazig University Hospitals. Science Journal of Public Health. Vol. 4, No. 6, 2016, pp. 506-516. doi: 10.11648/j.sjph.20160406.25

Received: November 29, 2016; Accepted: December 9, 2016; Published: January 10, 2017

\begin{abstract}
Background: Hypertension is an important risk factor for cardio vascular illness and mortality all over the world. The burdens of hypertension can be significantly controlled and reduced by lifestyle modification and treatment adherence. DASH diet is proved to reduce blood pressure either alone or combined with other life style modification. Aim of the Study is to evaluate effect of an educational intervention about Dietary Approach to Stop Hypertension (DASH) on Changing knowledge, attitude and blood pressure of hypertensive Patients Attending Zagazig University Hospitals. Methodology: this study was an interventional study carried out in cardiology outpatient clinic in Zagazig University Hospitals. The sample included 132 hypertensive patients chosen by systematic random sampling. The patients were divided into two equal groups 66 patients in the intervention group (G1) and 66 patients in the control group (G2). Data were collected through interviewing questionnaire which cover socio demographic characteristics, knowledge and attitude toward DASH diet. Results: The results showed that there was no significant difference between both groups in socio demographic characteristics, knowledge and attitude toward DASH before the intervention. There was significant improvement in all items of knowledge and attitude toward DASH in studied group 1 after application of health education sessions. There was significant reduction in blood pressure of studied group 1 after the intervention but there was no significant change in group 2. Conclusion: The health education program for hypertensive patients about DASH diet proved its success in improving their knowledge, attitude and reduction of their blood pressure level.
\end{abstract}

Keywords: Hypertension, DASH Diet, Life Style Modification, Health Education

\section{Introduction}

Hypertension is a persistent elevation of the systolic blood pressure at a level of $140 \mathrm{~mm}$ hg or higher and diastolic blood pressure at a level of $90 \mathrm{~mm} \mathrm{hg}$ or higher based on the average of two or more correct blood pressure measurement taken two or more contact with health care providers (WHO, 2015).

Poorly controlled hypertension is a significant worldwide public health concern because of its morbidity, mortality, and economic burden especially among older adults (Ogedegbe et al, 2013).
About one billion individuals or $26 \%$ of the adult population worldwide had hypertension (Park et al, 2011), while the prevalence of pre hypertension and hypertension in Egypt were estimated to be $57.2 \%$ and $17.6 \%$ respectively (Arafa and Ez-Elarab., 2011).

The healthy DASH (Dietary Approaches to Stop Hypertension) diet plan was developed to lower blood pressure without medication in research sponsored by the US National Institutes of Health. The first DASH diet research showed that it could lower blood pressure as well as the first 
line blood pressure medications, even with a sodium intake of $3300 \mathrm{mg} /$ day. Numerous studies have shown that the DASH diet reduces the risk of many diseases, including some kinds of cancer, stroke, heart disease, heart failure, kidney stones, and diabetes. It has been proven to be an effective way to lose weight and become healthier at the same time. (Heller, 2016)

DASH is a flexible and balanced eating plan that requires no special foods and instead provides daily and weekly nutritional goals. This plan recommends:

(1) Eating vegetables, fruits, and whole grains

(2) Including fat-free or low-fat dairy products, fish, poultry, beans, nuts, and vegetable oils

(3) Limiting foods that are high in saturated fat, such as fatty meats, full-fat dairy products, and tropical oils such as coconut, palm kernel, and palm oils

(4) Limiting sugar-sweetened beverages and sweets.(National Heart, Lung, and Blood Institute, 2015)

Beside its beneficial effect on blood pressure, DASH is designed to be a well-balanced diet for the all population. DASH is recommended by the United States Department of Agriculture (USDA) as an ideal eating plan for all American (USDA, 2010).

The DASH diet reduces systolic blood pressure by $6 \mathrm{~mm}$ $\mathrm{Hg}$ and diastolic blood pressure by $3 \mathrm{~mm} \mathrm{Hg}$ in patients with pre-hypertension (Systolic 120-139 mm Hg, diastolic 80-89 $\mathrm{mm} \mathrm{Hg}$ ). Those with hypertension (Systolic $\geq 140 \mathrm{~mm} \mathrm{Hg}$, diastolic $\geq 90 \mathrm{~mm} \mathrm{Hg}$ ) dropped by 11 and $6 \mathrm{~mm} \mathrm{Hg}$, respectively. These changes in blood pressure occurred with no changes in body weight (U.S. Department of Health and Human Services., 2006). It also reduces bad cholesterol (LDL) and the 10-year risk of heart attack (Chen et al, 2010).

The World Health Organization defined Health Education as any combination of learning experiences designed to help individuals and communities improve their health, by increasing their knowledge or influencing their attitudes (WHO, 2015).

\section{Pateints and Methods}

\subsection{Technical Design}

\subsubsection{Type of Study}

An interventional study

\subsubsection{Site of Study}

The present study was conducted at cardiology outpatient clinic in Zagazig University Hospitals.

\subsubsection{Sample Size and Sampling Technique}

The sample size was calculated using (open Epi) program assuming that the level of knowledge after health education (70\%) and level of knowledge before health education (50\%) (Habib et al., 2016), at confidence interval 95\% and at power $80 \%$, the estimated sample was 132 hypertensive patients. Patients will be chosen by systematic random sampling technique then divided into two equal groups 66 patients in the intervention group (G1) and 66 patients in the control group (G2). As the mean attendance of hypertensive patients is about 10 patients /day so each third patient will be chosen in the sample.

\subsubsection{Patients Included in Study}

132 hypertensive patients attending cardiology outpatient clinic in Zagazig University Hospitals.

Inclusion criteria:

Patients with hypertension (systolic $>140 \mathrm{~mm} \mathrm{hg}$, diastolic $>90 \mathrm{~mm} \mathrm{hg}$ ), Aged $>18$ years and attending cardiology outpatient clinic in Zagazig University Hospital regularly.

Exclusion criteria:

Age 18 years old or less, Patients with any physical or mental disability that hinder the intervention e.g., blindness or deafness, Patients with diagnosed secondary hypertension (renal or endocrinal), Patients with isolated systolic hypertension.

\subsubsection{Data Collection Tools}

The intervention group received pre test to assess their knowledge and attitude before the health education sessions then they received post test after two months of the intervention to evaluate the effect of the health education sessions, while the control group received pre test to assess their knowledge and attitude then they received post test after two months without any intervention.

These data were collected by using:

(1) An interviewing Arabic pre-test questionnaire which was designed by (Habib et al., 2016) for hypertensive patients.

It included the following items:

(A) Socio demographic characteristics of the patients: such as age, gender, education of husband and wife... etc.

Social class was scored as follows: education and cultural domain for both husband \& wife (score $=30$ ), family domain (score $=4$ ), economic domain (score $=3$ ), occupational domain (for both husband \& wife) (score $=10$ ), home domain ( score $=1)$, health care domain $($ score $=5$ ) and total score will be 53. Socioeconomic level was then classified into Very low: $<13.5$, Low: $13.5-<27$, Middle: $27-<40$ and High: $\geq 40$ (El-Gilany, 2012)

(B) Questions to test the knowledge of the patient and include the following points:

1) General knowledge about DASH:- hearing about DASH, Importance of having lots of fruits and vegetables, Suitable type of dairy products, Effect of excess salt and salty foods on blood pressure, The best type of bread, Does excess fats and fatty foods effect blood pressure, Suitable type of fats, The best type of oil, Effect of exercise on blood pressure and Effect of smoking on blood pressure

2) Knowledge about serving size in DASH:- The suitable serving size of fruits and vegetables, dairy products, meat, poultry, fish, seeds, dry beans, grains, and sweets. 
(C) Questions to test the attitude towards DASH:Following the DASH plan lower blood pressure, Limiting salt intake control blood pressure, Having fruits instead of sweets as a desert and Stop smoking and making exercise help to lower blood pressure.

Scoring of patient knowledge: in the questions about the suitable serving size, all answers are correct so:-Getting less than or equal to $50 \%$ of answers or don't know is considered wrong.

Getting more than $50 \%$ of answers is considered right. *In other questions The right answer equals 1 The wrong answer equals 0 .Maximum score equal 17, Minimum score equal 0. Good knowledge $>50 \%$ while, Poor knowledge $\leq 50 \%$.

Scoring of attitude questions:

Answers were: Agree equal 1 degree, Disagree equal 0 degree, Maximum score equal 4,Minimum score equal 0 , Good score $>50 \%$ and Poor score $\leq 50 \%$

(2) The same pre-test questionnaire was used as a post test to assess knowledge and attitude of the intervention group (G1) after health education sessions, while the control group (G2) completed this post test without any intervention.

(3) Measuring blood pressure.

Blood pressure was measured before the intervention and two month after it for both groups. The blood pressure was measured by mercury sphygmomanometer. Reading blood pressure by auscultation is considered the gold standard by the Heart, Lung and Blood Institute of the National Institute of Health (NIH). The blood pressure was measured while patients were in seated position with flexed arm, the flexed elbow should be at the level of the heart and supported and if the patient is anxious, wait a few minutes before taking the pressure. (Clark et al., 2012).

(4) Health education message.

The hypertensive patients included in the intervention group (G1) were given educational sessions to improve knowledge and attitude of the patient toward DASH: Definition of dietary approach to stop hypertension, importance of DASH diet, DASH eating plan and other life style modification needed to control hypertension. The educational message was conducted by the researcher through personal interview in health education sessions. Each session took about 20 minutes and was held three times weekly in the cardiology outpatient clinic. The message was facilitated by uses of posters and booklets which contain the most important items in the health education message.

\subsection{Operational Design}

\subsubsection{Pilot Study}

Before starting the actual field study, a pilot study was carried on 10 hypertensive patients to estimate the level of patient's knowledge because of absence of Egyptian reference and to test the questionnaire with the most appropriate terms. It also helped to estimate time needed for data collection, and detect the obstacles of the study. The patients included in pilot study were not included in the main sample.

\subsubsection{Data Collection and Work Field}

(1) Data collection and health education sessions were done between March 2016 to september 2016 with average two months lapse between pre and post test.

(2) The researcher collected the pre-test questionnaires by meeting the hypertensive patients attending cardiology outpatient clinic in Zagazig University Hospital after explaining to them the objectives of the study.

(3) The blood pressure was measured by mercury sphygmomanometer before and after the intervention.

(4) Health education sessions provided for hypertensive patients in the cardiology outpatient clinic (group 1).

(5) The post-test questionnaires were collected to assess knowledge and attitude after health education sessions for both groups.

\subsubsection{Administrative Design and Ethical Aspects}

The necessary official permissions were obtained: Approval obtained for performing the study from Zagazig University Hospital manager and the head of cardiology department after explaining the purpose of the study to them. Approval from the Institutional Review Board of Faculty of Medicine, Zagazig University. An informed consent was obtained from hypertensive patients involved in the study after explaining purpose to them. The study group was not exposed to any harm or risk and their data were confidential.

\subsubsection{Data Management}

Collected data were presented in tables and suitable graphs and analyzed by computer software (statistical package SPSS version 16) using appropriate statistical methods. Frequencies, means and standard deviation were used to summarize the data. Categorical data were compared by using chi square test. MC 'Nemar test was used to compare pre and post test. Student $t$ test was used to compare two independent quantitative data. Paired t- test was used to compare paired quantitative data. Probability was considered significant if $\mathrm{P}$. value is less than or equal to 0.05 .

\section{Results}

As regard comparison between the two groups as regard socio demographic characteristics (Table 1): there is no statistically significant difference between socio demographic characteristics of both groups.

As regard Comparison between pre-tests of studied groups about general knowledge of DASH (Table 2). It shows that there is no statistical significant difference between pretests of studied groups about general knowledge of DASH. 
Table 1. Comparison between the two groups as regard socio demographic characteristics.

\begin{tabular}{|c|c|c|c|c|c|c|}
\hline \multirow{2}{*}{ Variables } & \multicolumn{2}{|c|}{$\operatorname{group}(1)$} & \multicolumn{2}{|c|}{ group (2) } & \multirow{2}{*}{$X^{2}$} & \multirow{2}{*}{$\mathbf{P}$} \\
\hline & No (66) & $\%$ & No (66) & $\%$ & & \\
\hline \multicolumn{7}{|l|}{ Age } \\
\hline $45-<60$ & 35 & 53.0 & 29 & 43.9 & \multirow[t]{2}{*}{1.095} & \multirow[t]{2}{*}{0.295} \\
\hline $60-75$ & 31 & 47.0 & 37 & 56.1 & & \\
\hline \multicolumn{7}{|l|}{ Sex } \\
\hline Male & 25 & 37.9 & 33 & 50.0 & \multirow[t]{2}{*}{1.107} & \multirow[t]{2}{*}{0.293} \\
\hline Female & 41 & 62.1 & 33 & 50.0 & & \\
\hline \multicolumn{7}{|c|}{ Education of husband } \\
\hline Illiterate & 19 & 28.8 & 26 & 39.4 & \multirow{5}{*}{8.093} & \multirow{5}{*}{0.088} \\
\hline Read and write & 15 & 22.7 & 21 & 31.8 & & \\
\hline Essential education & 4 & 6.1 & 0 & 0.0 & & \\
\hline Secondary & 26 & 39.4 & 17 & 25.8 & & \\
\hline High & 2 & 3.0 & 2 & 3.0 & & \\
\hline \multicolumn{7}{|l|}{ Education of wife } \\
\hline Illiterate & 21 & 31.8 & 30 & 45.5 & \multirow{5}{*}{8.604} & \multirow{5}{*}{0.072} \\
\hline Read and write & 27 & 40.9 & 29 & 43.9 & & \\
\hline Essential education & 4 & 6.1 & 0 & 0.0 & & \\
\hline Secondary & 12 & 18.2 & 5 & 7.6 & & \\
\hline High & 2 & 3.0 & 2 & 3.0 & & \\
\hline \multicolumn{7}{|c|}{ Occupation of husband } \\
\hline Not working & 27 & 40.9 & 21 & 31.8 & \multirow{5}{*}{7.655} & \multirow{5}{*}{0.105} \\
\hline Farmer & 7 & 10.6 & 13 & 19.7 & & \\
\hline Worker & 16 & 24.2 & 24 & 36.4 & & \\
\hline Clerk & 14 & 21.2 & 8 & 12.1 & & \\
\hline Business man & 2 & 3.0 & 0 & 0 & & \\
\hline \multicolumn{7}{|l|}{ Occupation of wife } \\
\hline Not working & 59 & 89.4 & 63 & 95.5 & \multirow[t]{2}{*}{1.467} & \multirow[t]{2}{*}{0.226} \\
\hline Clerk & 7 & 10.6 & 3 & 4.5 & & \\
\hline \multicolumn{7}{|l|}{ Residence } \\
\hline Urban & 19 & 28.8 & 27 & 40.9 & \multirow[t]{2}{*}{2.182} & \multirow[t]{2}{*}{0.139} \\
\hline Rural & 47 & 71.2 & 39 & 59.1 & & \\
\hline Social class & & & & & & \\
\hline Low & 55 & 83.3 & 62 & 93.9 & 3.308 & 0.068 \\
\hline Moderate & 11 & 16.7 & 4 & 6.1 & & \\
\hline Smoking & & & & & & \\
\hline Smoker & 43 & 65.2 & 44 & 66.7 & $\cap \cap 6$ & 0071 \\
\hline Ex-smoker & 11 & 18.2 & 10 & 15.2 & 0.06 & 0.911 \\
\hline Non smoker & 12 & 18.2 & 12 & 18.3 & & \\
\hline
\end{tabular}

Table 2. Comparison between pre-tests of studied groups about general knowledge of DASH.

\begin{tabular}{|c|c|c|c|c|c|c|}
\hline \multirow{2}{*}{ Variables } & \multicolumn{2}{|c|}{ Pre-test G1 } & \multicolumn{2}{|c|}{ Pre-test G2 } & \multirow{2}{*}{$X^{2}$} & \multirow{2}{*}{$\mathbf{P}$} \\
\hline & No (66) & $\%$ & No (66) & $\%$ & & \\
\hline \multicolumn{7}{|l|}{ Hearing about DASH } \\
\hline - Yes & 42 & 63.6 & 40 & 60.6 & 0.125 & 0.723 \\
\hline - $\mathrm{NO}$ & 24 & 36.4 & 26 & 39.4 & & \\
\hline \multicolumn{7}{|c|}{ Importance of having lots of fruits and vegetables } \\
\hline - Yes & 34 & 51.5 & 44 & 66.7 & 3.075 & 0.079 \\
\hline - $\mathrm{NO}$ & 32 & 48.5 & 22 & 33.3 & & \\
\hline \multicolumn{7}{|c|}{ Suitable type of dairy products } \\
\hline - Full cream & 40 & 60.6 & 30 & 45.5 & 3.030 & 0.082 \\
\hline - Skimmed or low cream & 26 & 39.4 & 36 & 54.5 & & \\
\hline \multicolumn{7}{|c|}{ Effect of excess salt and salty foods on blood pressure } \\
\hline - Elevate & 52 & 78.8 & 59 & 89.4 & 2.418 & 0.120 \\
\hline - Lower & 14 & 21.2 & 7 & 10.6 & & \\
\hline \multicolumn{7}{|l|}{ The best type of bread } \\
\hline - White bread & 26 & 39.4 & 17 & 25.8 & 2.948 & 0.086 \\
\hline - Whole grain bread & 40 & 60.6 & 49 & 74.2 & & \\
\hline \multicolumn{7}{|c|}{ Does excess fats and fatty foods effect blood pressure } \\
\hline - yes & 48 & 72.7 & 56 & 81.8 & 2.640 & 0.104 \\
\hline - No & 18 & 27.3 & 10 & 18.2 & & \\
\hline \multicolumn{7}{|l|}{ Suitable type of fats } \\
\hline - Oil & 44 & 63.6 & 45 & 68.2 & 0.03 & 0.853 \\
\hline - Butter or margarine & 22 & 36.4 & 21 & 31.8 & & \\
\hline $\begin{array}{l}\text { The best type of oil } \\
\text { - Olive }\end{array}$ & 6 & 9.1 & 4 & 6.1 & 0.70 & 0.403 \\
\hline
\end{tabular}




\begin{tabular}{|c|c|c|c|c|c|c|}
\hline \multirow{2}{*}{ Variables } & \multicolumn{2}{|c|}{ Pre-test G1 } & \multicolumn{2}{|c|}{ Pre-test G2 } & \multirow{2}{*}{$X^{2}$} & \multirow{2}{*}{$\mathbf{P}$} \\
\hline & No (66) & $\%$ & No (66) & $\%$ & & \\
\hline - Corn or sun flower & 60 & 90.9 & 62 & 93.9 & & \\
\hline \multicolumn{7}{|l|}{ Effect of exercise on blood pressure } \\
\hline - Lower & $\begin{array}{l}28 \\
38\end{array}$ & $\begin{array}{l}42.4 \\
57.6\end{array}$ & 36 & $\begin{array}{l}54.5 \\
45.5\end{array}$ & 1.956 & 0.162 \\
\hline \multicolumn{7}{|l|}{ Effect of smoking on blood pressure } \\
\hline $\begin{array}{l}\text { - Elevate } \\
\text { - Lower or does not effect }\end{array}$ & $\begin{array}{l}26 \\
40\end{array}$ & $\begin{array}{l}39.4 \\
60.6\end{array}$ & $\begin{array}{l}34 \\
32\end{array}$ & $\begin{array}{l}51.5 \\
48.5\end{array}$ & 1.985 & 0.159 \\
\hline
\end{tabular}

As regard Comparison between pre-tests of the studied groups about knowledge of serving size in DASH (Table 3). There is no statistical significant difference between pretests of studied groups' about serving size in DASH.

Table 3. Comparison between pre-tests of the studied groups about knowledge of serving size in DASH.

\begin{tabular}{|c|c|c|c|c|c|c|}
\hline \multirow{2}{*}{ Variables } & \multicolumn{2}{|c|}{ Pre-test G1 } & \multicolumn{2}{|c|}{ Pre-test G2 } & \multirow{2}{*}{$X^{2}$} & \multirow{2}{*}{$\mathbf{P}$} \\
\hline & No (66) & $\%$ & No (66) & $\%$ & & \\
\hline \multicolumn{7}{|c|}{ Suitable serving size of vegetables } \\
\hline - Adequate knowledge & 27 & 40.9 & 26 & 39.4 & \multirow{3}{*}{0.03} & \multirow{3}{*}{0.859} \\
\hline - Inadequate knowledge & 39 & 59.1 & 40 & 60.6 & & \\
\hline \multicolumn{5}{|c|}{ Suitable serving size of fruits } & & \\
\hline - Adequate knowledge & 27 & 40.9 & 29 & 43.9 & \multirow{3}{*}{0.129} & \multirow{2}{*}{0.719} \\
\hline - Inadequate knowledge & 39 & 59.1 & 37 & 56.1 & & \\
\hline \multicolumn{6}{|c|}{ Suitable serving size of dairy products } & \\
\hline - Adequate knowledge & 35 & 53.0 & 45 & 68.2 & \multirow[t]{2}{*}{3.075} & \multirow[t]{2}{*}{0.079} \\
\hline - Inadequate knowledge & 31 & 47.0 & 21 & 31.8 & & \\
\hline \multicolumn{7}{|c|}{ Suitable serving size of meat, poultry and fish } \\
\hline - Adequate knowledge & 45 & 68.2 & 53 & 80.3 & \multirow{2}{*}{2.296} & \multirow{2}{*}{0.129} \\
\hline - Inadequate knowledge & 21 & 31.8 & 13 & 19.7 & & \\
\hline \multicolumn{7}{|c|}{ Suitable serving size of seeds and dry beans } \\
\hline - Adequate knowledge & 15 & 22.7 & 13 & 19.7 & \multirow{2}{*}{0.218} & \multirow{2}{*}{0.640} \\
\hline - Inadequate knowledge & 51 & 77.3 & 53 & 80.3 & & \\
\hline \multicolumn{7}{|c|}{ Suitable serving size of grains } \\
\hline - Adequate knowledge & 34 & 51.5 & 27 & 40.9 & \multirow{2}{*}{1.128} & \multirow{2}{*}{0.288} \\
\hline - Inadequate knowledge & 32 & 48.5 & 39 & 59.1 & & \\
\hline \multicolumn{7}{|c|}{ Suitable serving size of sweets } \\
\hline - Adequate knowledge & 19 & 28.8 & 16 & 24.2 & \multirow{2}{*}{0.35} & \multirow{2}{*}{0.554} \\
\hline - Inadequate knowledge & 47 & 71.2 & 50 & 75.8 & & \\
\hline
\end{tabular}

As regard Comparison between pre-test of studied group (1) and pre-test of studied group (2) about attitude towards DASH (Table 4). There is no statistically significant difference between the two groups as regard attitude towards DASH.

Table 4. Comparison between pre-test of studied groups about attitude towards DASH.

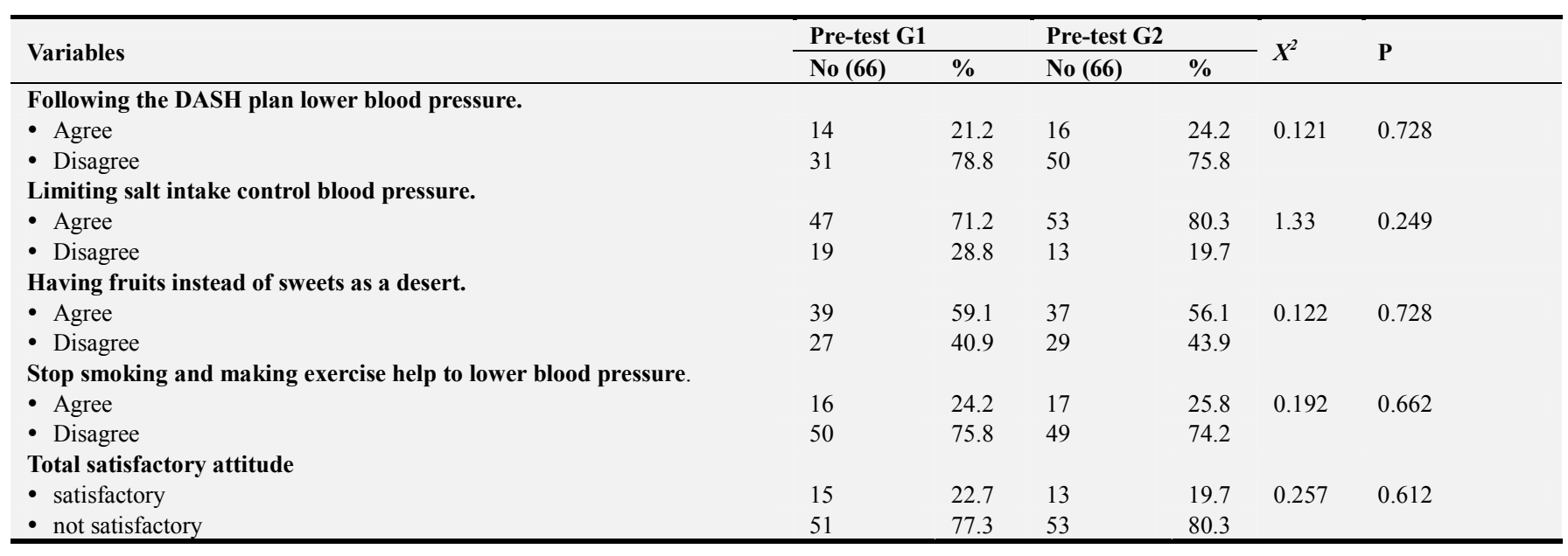

Regarding Changes of general knowledge about DASH among the studied group (1) \& group (2) (table 5). It shows statistically significant improvement in all items concerning general knowledge about DASH after health education especially the best type of oil (olive oil) which was improved from $9.1 \%$ in pre test to $54.5 \%$ in post test followed by the importance of having lots of fruits and vegetables which was improved from $51.5 \%$ in pre test to $92.4 \%$ in post test. While, there is no statistically significant change in all items concerning general knowledge about DASH among studied group (2). 
Table 5. Changes of general knowledge about DASH among the studied groups after two months from intervention among group (1) and from pre test among group (2).

\begin{tabular}{|c|c|c|c|c|c|c|c|c|c|c|}
\hline \multirow{3}{*}{ Variables } & \multicolumn{5}{|c|}{ group (1) } & \multicolumn{5}{|c|}{ group (2) } \\
\hline & \multicolumn{2}{|l|}{ Pre-test } & \multicolumn{2}{|c|}{ Post- test } & \multirow{2}{*}{$\mathbf{P}^{*}$} & \multicolumn{2}{|l|}{ Pre-test } & \multicolumn{2}{|c|}{ Post- test } & \multirow{2}{*}{$\mathbf{P}^{*}$} \\
\hline & No (66) & $\%$ & No (66) & $\%$ & & No (66) & $\%$ & No (66) & $\%$ & \\
\hline \multicolumn{11}{|c|}{ Importance of having lots of fruits and vegetables } \\
\hline - Yes & 34 & 51.5 & 61 & 92.4 & \multirow[t]{2}{*}{$<0.001$} & 44 & 66.7 & 46 & 69.7 & \multirow[t]{2}{*}{0.708} \\
\hline - No & 32 & 48.5 & 5 & 7.6 & & 22 & 33.3 & 20 & 30.3 & \\
\hline \multicolumn{11}{|c|}{ Suitable type of dairy products } \\
\hline - Full cream & 40 & 60.6 & 18 & 27.3 & \multirow[t]{2}{*}{$<0.001$} & 30 & 45.5 & 23 & 34.8 & \multirow[t]{2}{*}{0.629} \\
\hline - Skimmed or low cream & 26 & 39.4 & 48 & 62.7 & & 36 & 54.5 & 43 & 65.2 & \\
\hline \multicolumn{11}{|c|}{ Effect of excess salt and salty foods on blood pressure } \\
\hline - Elevate & 52 & 78.8 & 65 & 98.5 & \multirow{2}{*}{0.001} & 59 & 89.4 & 61 & 92.4 & \multirow[t]{2}{*}{0.508} \\
\hline - Lower & 14 & 21.2 & 1 & 1.5 & & 7 & 10.6 & 5 & 7.6 & \\
\hline \multicolumn{11}{|l|}{ The best type of bread } \\
\hline - White bread & 26 & 39.4 & 10 & 15.2 & \multirow[t]{2}{*}{$<0.001$} & 17 & 25.8 & 15 & 22.7 & \multirow[t]{2}{*}{0.685} \\
\hline - Whole grain bread & 40 & 60.6 & 56 & 84.8 & & 49 & 74.2 & 51 & 77.3 & \\
\hline \multicolumn{11}{|c|}{ does excess fats and fatty foods Effect blood pressure } \\
\hline - yes & 48 & 72.7 & 64 & 97.0 & \multirow{2}{*}{$<0.001$} & 56 & 81.8 & 57 & 86.3 & \multirow[t]{2}{*}{0.687} \\
\hline - No & 18 & 27.3 & 2 & 3.0 & & 10 & 18.2 & 9 & 15.2 & \\
\hline \multicolumn{11}{|l|}{ Suitable type of fats } \\
\hline - Oil & 44 & 63.6 & 62 & 93.9 & \multirow[t]{2}{*}{$<0.001$} & 45 & 68.2 & 46 & 69.7 & \multirow[t]{2}{*}{0.851} \\
\hline - Butter or margarine & 22 & 36.4 & 4 & 6.1 & & 21 & 31.8 & 20 & 30.3 & \\
\hline \multicolumn{11}{|l|}{ The best type of oil } \\
\hline - Olive & 6 & 9.1 & 36 & 54.5 & \multirow[t]{2}{*}{$<0.001$} & 4 & 6.1 & 5 & 7.6 & 0.729 \\
\hline - Corn or sun flower & 60 & 90.9 & 30 & 45.5 & & 62 & 93.9 & 61 & 92.4 & \\
\hline Effect of exercise on blood & & & & & & & & & & \\
\hline - Lower & 28 & 42.4 & 48 & 62.7 & $<0.001$ & 36 & 54.5 & 34 & 51.5 & 0.727 \\
\hline - Elevate or does not effect & 38 & 57.6 & 18 & 37.3 & & 30 & 45.5 & 32 & 48.5 & \\
\hline Effect of smoking on blood & & & & & & & & & & \\
\hline - Elevate & 26 & 39.4 & 44 & 66.7 & $<0.001$ & 34 & 51.5 & 33 & 50.0 & 0.862 \\
\hline - Lower or does not effect & 40 & 60.6 & 22 & 33.3 & & 32 & 48.5 & 33 & 50.0 & \\
\hline
\end{tabular}

*N.B: McNemar test of significance.

As regard Changes of knowledge about serving size in DASH among group 1\& group 2 (Table 6). There is statistically significant improvement in all items concerning knowledge about serving size in DASH after application of health education sessions among the studied group 1 especially adequate knowledge about suitable serving size of seeds and dry beans which was improved from $22.7 \%$ in pre test to $60.6 \%$ in post. While, there is no statistically significant change in all items concerning knowledge about serving size in DASH among the studied group (2).

Table 6. Changes of knowledge about serving size in DASH among studied groups after two months from intervention among group (1) and from pre test among group (2).

\begin{tabular}{|c|c|c|c|c|c|c|c|c|c|c|}
\hline \multirow{3}{*}{ Variables } & \multicolumn{5}{|c|}{ group (1) } & \multicolumn{5}{|c|}{ group (2) } \\
\hline & \multicolumn{2}{|l|}{ Pre-test } & \multicolumn{2}{|c|}{ Post- test } & \multirow{2}{*}{$-\mathbf{P}^{*}$} & \multicolumn{2}{|l|}{ Pre-test } & \multicolumn{2}{|c|}{ Post- test } & \multirow{2}{*}{$\mathbf{P}^{*}$} \\
\hline & No (66) & $\%$ & No (66) & $\%$ & & No (66) & $\%$ & No (66) & $\%$ & \\
\hline \multicolumn{11}{|l|}{ Suitable serving size of vegetables } \\
\hline - Adequate knowledge & 27 & 40.9 & 44 & 66.7 & \multirow[t]{2}{*}{$<0.001$} & 26 & 39.4 & 27 & 40.9 & \multirow[t]{2}{*}{0.859} \\
\hline - Inadequate knowledge & 39 & 59.1 & 22 & 33.3 & & 40 & 60.6 & 39 & 59.1 & \\
\hline \multicolumn{11}{|l|}{ Suitable serving size of fruits } \\
\hline - Inadequate knowledge & 39 & 59.1 & 20 & 30.3 & $<0.001$ & 37 & 56.1 & 36 & 54.5 & 0.861 \\
\hline \multicolumn{11}{|l|}{ Suitable serving size of dairy products } \\
\hline - Adequate knowledge & 35 & 53.0 & 52 & 78.8 & \multirow{2}{*}{$<0.001$} & 45 & 68.2 & 43 & 65.2 & \multirow[t]{2}{*}{0.711} \\
\hline - Inadequate knowledge & 31 & 47.0 & 14 & 21.2 & & 21 & 31.8 & 23 & 34.8 & \\
\hline \multicolumn{11}{|l|}{ Suitable serving size of meat, poultry and fish } \\
\hline - Adequate knowledge & 45 & 68.2 & 60 & 90.9 & \multirow{2}{*}{$<0.001$} & 53 & 80.3 & 52 & 78.8 & \multirow[t]{2}{*}{0.681} \\
\hline - inadequate knowledge & 21 & 31.8 & 6 & 9.1 & & 13 & 19.7 & 14 & 21.2 & \\
\hline - Adequate knowledge & 15 & 22.7 & 40 & 60.6 & $<0.001$ & 13 & 19.7 & 12 & 18.2 & 0.754 \\
\hline
\end{tabular}




\begin{tabular}{|c|c|c|c|c|c|c|c|c|c|c|}
\hline \multirow{3}{*}{ Variables } & \multicolumn{5}{|c|}{ group (1) } & \multicolumn{5}{|l|}{ group (2) } \\
\hline & \multicolumn{2}{|l|}{ Pre-test } & \multicolumn{2}{|c|}{ Post- test } & \multirow{2}{*}{$\mathbf{P} *$} & \multicolumn{2}{|l|}{ Pre-test } & \multicolumn{2}{|c|}{ Post- test } & \multirow{2}{*}{$\mathbf{P}^{*}$} \\
\hline & No (66) & $\%$ & No (66) & $\%$ & & No (66) & $\%$ & No (66) & $\%$ & \\
\hline \multicolumn{11}{|c|}{ Suitable serving size of grains } \\
\hline - Adequate knowledge & 34 & 51.5 & 52 & 78.8 & $<0.001$ & 27 & 40.9 & 26 & 39.4 & 0.500 \\
\hline - Inadequate knowledge & 32 & 48.5 & 14 & 21.2 & & 39 & 59.1 & 40 & 60.6 & \\
\hline - Adequate knowledge & 19 & 28.8 & 38 & 57.6 & $<0.001$ & 16 & 24.2 & 18 & 27.3 & 0.727 \\
\hline - Inadequate knowledge & 47 & 71.2 & 28 & 42.4 & & 50 & 75.8 & 48 & 72.7 & \\
\hline
\end{tabular}

N.B: McNemar test of significance.

Regarding changes of attitude towards DASH plan among group 1 and group 2 of the studied sample (Table 7). There is statistically significant improvement in all items concerning attitude towards DASH in group (1) after application of health education sessions especially their positive attitude toward stop smoking and making exercise help to lower blood pressure which was improved from $22.7 \%$ in pre test to $62.1 \%$ in post test. While, there is no statistically significant change in all items concerning attitude towards DASH in group (2).

Table 7. Changes of attitude towards DASH plan among studied groups after two months from intervention among group (1) and from pre test among group (2).

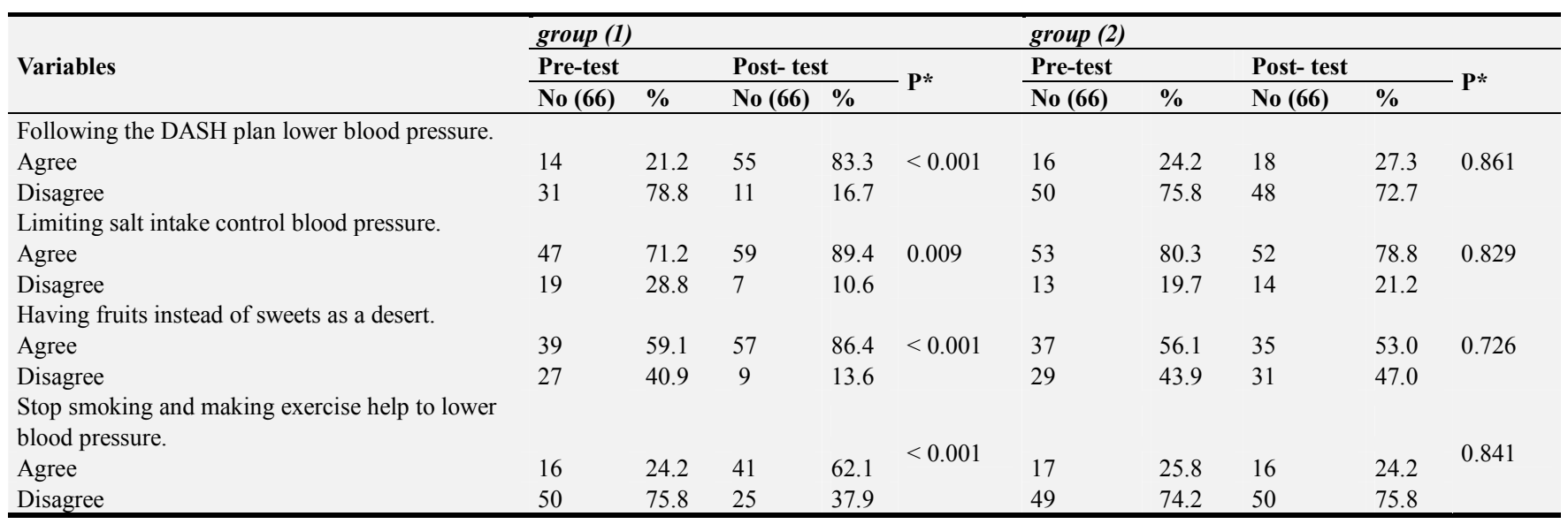

As regard changes of total knowledge and attitude among the studied group (1) and group (2) (Figure 1). It shows statistically significant improvement in both total knowledge scores from $40.9 \%$ in pre test to $72.7 \%$ in post test and total attitude scores from $22.7 \%$ to $83.3 \%$ after application of health education sessions among the studied group (1). While, there is no statistically significant difference in both total knowledge scores and total attitude scores among group (2) between pre and post test.

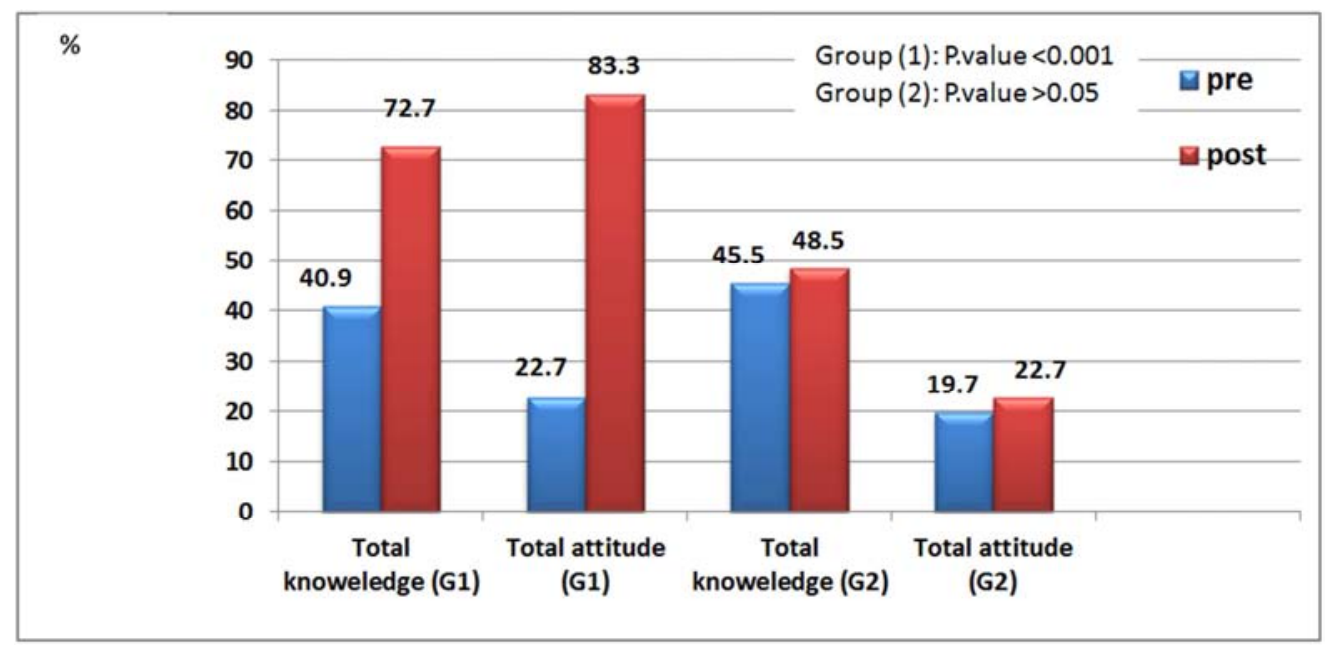

Figure 1. Changes of total knowledge and attitude among studied groups after two months from intervention among group (1) and from pre test among group (2). 
Regarding Comparison between blood pressure levels of studied group (1) and studied group (2) before the intervention Table (8). There is no statistical significant difference in both systolic and diastolic blood pressure between the two groups.

Table 8. Comparison between blood pressure levels of studied groups at the beginning of the study.

\begin{tabular}{lllll}
\hline Blood pressure & Group (1) & Group (2) & t test & P \\
\hline Systolic mean \pm SD & $144.7 \pm 12.7$ & $145.1 \pm 12.2$ & 0.7 & 0.485 \\
Diastolic mean \pm SD & $93.8 \pm 7.9$ & $94.2 \pm 6.9$ & 1.64 & 0.103 \\
\hline
\end{tabular}

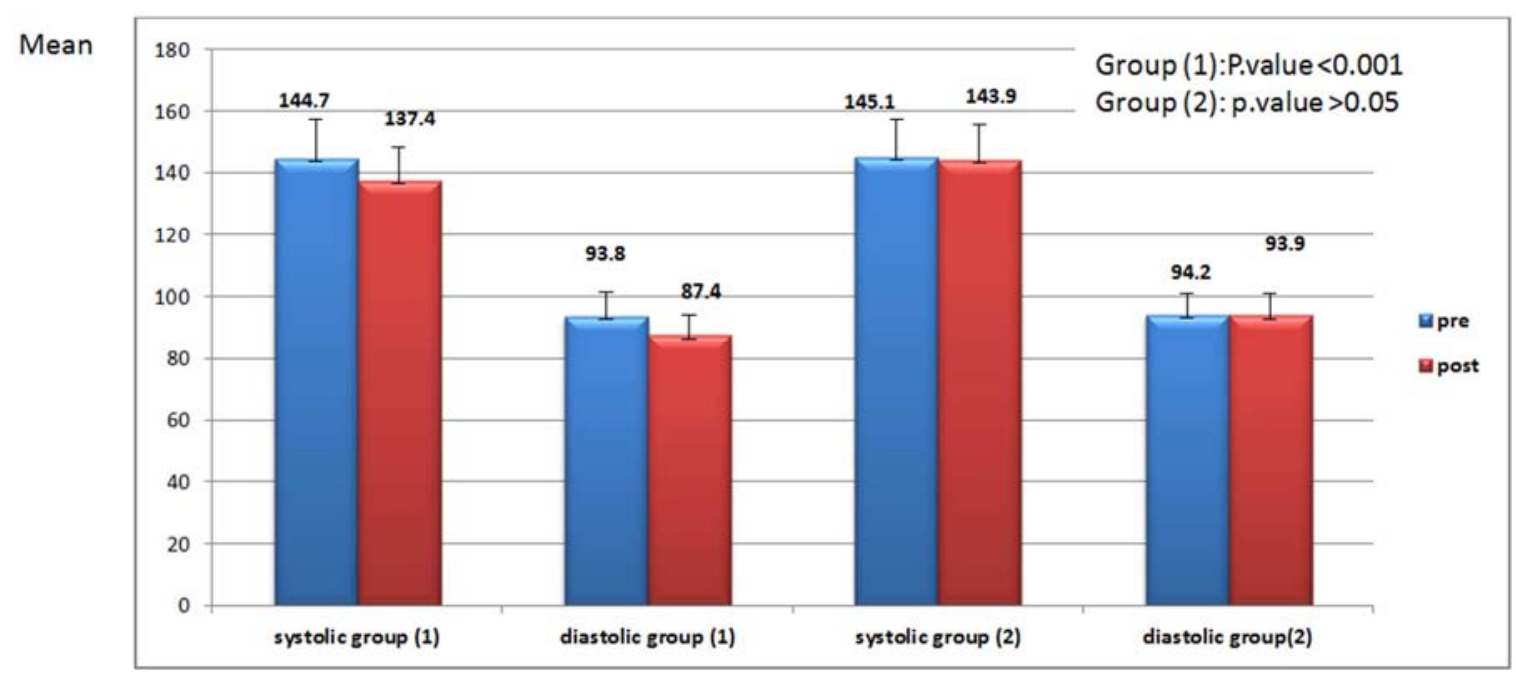

Figure 2. Changes of blood pressure among the studied groups after two months from intervention among group (1) and from pre test among group (2).

As regard Changes of blood pressure among the studied group (1) \& group (2) (Figure 2). There is statistically significant reduction in both systolic and diastolic blood pressure in group (1) after application of health education intervention. The mean systolic blood pressure had decreased from 144.7 before intervention to 137.4 after intervention and mean diastolic blood pressure had decreased from 93.8 before intervention to 87.4 after intervention. While there is no statistical significant change in both systolic and diastolic blood pressure among group (2).

\section{Discussion}

\subsection{Assessment of the Patient's Knowledge}

In the present study there was no significant difference between the intervention and the control group neither in general knowledge about DASH nor in knowledge about the serving size in DASH before the intervention (table 2 and table 3). This may be due to most of patients were of relatively the same social class and there was no significant difference in their educational level.

This result was consistent with Jeng, (2011) Al-Weedy et al., (2014) and Kamran et al., (2015) who studied the effect of DASH educational intervention on knowledge, selfefficacy and dietary practice, there was no significant difference in knowledge between intervention and control group before the intervention. Also, it was consistent with the results of Habib et al., (2016) who revealed no significant difference between the intervention and the control group neither in general knowledge about DASH nor in knowledge about the serving size in DASH before the intervention.

\subsection{Effect of Health Education on Patient's Knowledge}

Knowledge plays an important role in the nutrition of hypertension patients. (Ford et al, 2009). It is well known that health education can result in lifestyle modifications and improve blood pressure control in hypertensive patients (Shaw and Bosworth, 2012).

In the present study the application of nutritional education in the intervention group (1) led to significant improvement in all items of general knowledge, knowledge about serving size and total knowledge about DASH diet (table 5, 6 figure $1)$.

These results were consistent with the results of the study by Magadza et al., (2009), Jeng, (2011) and the study by Pandey et al., (2013) which indicated a significant increase in nutritional knowledge of the patients after the intervention.

This result also agreed with Harrison, (2014) who tested the efficacy of educational intervention about DASH in classrooms versus internet education. Seung-Hye and ChoiKwon (2014) also reported that one educational session increased dietary knowledge but dietary self-efficacy and DASH diet compliance did not increase significantly. Also, this study was consistent with result of Habib et al., 2016 who revealed significant improvement of knowledge of interventional group regarding general knowledge about DASH and knowledge about the serving size of DASH after the intervention done on hypertensive Patients Attending Dierb Negm hospital.

Assessment of knowledge of the control group (2) after two months of application of the pretest revealed that there was no significant change neither in general knowledge about 
DASH nor in knowledge about serving size and total knowledge (table 5, 6 figure 1). These results may be explained by

- They were not given health education sessions about DASH by their doctors.

- Absence of mass media campaigns about DASH during the period of the study.

The studies of Jeng, (2011), Al-Wehedyet et al., (2014) and Habib et al., 2016 also reported that there was no significant change in knowledge of the control group.

\subsection{Assessment of the Patient's Attitude}

This study found that only $(22.7 \%)$ had satisfactory attitude among group (1) and (19.7\%) among group (2) (table 4). This result may be attributed to lack of awareness in hypertensive patients about healthy life style. The low level of awareness may be explained by:

- Lack of mass media campaigns about DASH eating plan and other life style modifications.

- Doctors ignore nutritional education as a part of hypertension management.

- Doctors don't educate patients how to follow the DASH eating plan.

In India, Mahajan et al (2012) revealed that the majority of hypertensive patients had unsatisfactory attitude. Also, it was relatively consistant with results of Habib et al., 2016 who revealed that $13.6 \%$ of studied population had satisfactory attitude before health education intervention.

On the contrary Sabouhi et al (2011) reported that patient's attitude was good (58.2\%).

The present study revealed that there was no significant difference in patient's attitude between the two groups before intervention (table 4). This may be attributed to homogeneity of the studied sample in socio demographic characters and level of knowledge.

This study showed that $21.2 \%$ of the patients agreed that DASH diet is important in hypertension management (table 4). This result was going with Mahajan et al (2012) stated that only $5 \%$ of patients agreed that DASH diet is important in hypertension management. While, it was in contrary with Sabouhi et al (2011). Tadevosyan (2013) and Rakumakoe (2011) reported better attitude (70\% and 96\% respectively) and Habib et al., 2016 revealed $50 \%$ of the patients agreed that DASH diet is important in hypertension management.

About $71.2 \%$ of the participants agreed that reducing salt is important in controlling blood pressure (table 4). This result may be attributed to good knowledge of patients about salt effect on blood pressure. This result was consistent with Mahajan et al (2012) and Habib et al., 2016 who revealed $71 \%$ of the participants agreed that reducing salt is important in controlling blood pressure. Other studies conducted by Parmar et al (2014) and Tadevosyan (2013)reported that (40\% and $51 \%$ respectively) of patients agreed that reducing salt is important in controlling blood pressure.

The present study showed that $59.1 \%$ of the patients agreed that fruits are better than sweets as a desert and $24.2 \%$ of the sample agreed that exercise and stop smoking are important in hypertension management (table 4). This result was consistent with Mahajan et al (2012) and Habib et al., 2016. Parmar et al (2014) and Rakumakoe (2011) reported better attitude $(45.2 \%, 82 \%$ respectively). This discrepancy is due to lake of knowledge about the importance of exercise and stop smoking in controlling hypertension, old age of the patients and lack of facilities to exercise.

\subsection{Effect of Health Education on Patient's Attitude}

In the present study the application of nutritional education in the intervention group, lead to significant improvement in patient's positive attitude (table 7 and figure 1). This result may be attributed to improvement of patient's knowledge after health education sessions.

This result was consistent with Mahajan et al (2012), Sabouhi et al (2011) and Habib et al., 2016.

There was no significant change in attitude of patients in the control group two months after application of the pretest (table 7 and figure 1) because there was no change in patient's knowledge. This result was consistent with Habib et al., 2016.

\subsection{Changes of Blood Pressure in Intervention and Control Group}

In this study there was no significant difference in the mean of systolic and diastolic blood pressure between the two groups before the intervention (table 8) because of the homogeneity of the sample in socio demographic characters and the level of knowledge.

After the intervention, there was significant difference in the mean of systolic and diastolic blood pressure in the intervention group (G1) (figure 2). The mean systolic blood pressure had decreased from 144.7 before intervention to 137.4 after intervention and mean diastolic blood pressure had decreased from 93.8 before intervention to 87.4 after intervention. These results were consistent with Appel et al (1997) and Al-Wehedyet al (2014). Also, it was consistent with Habib et al., 2016 who revealed decrease of mean systolic blood pressure from 142.7 before intervention to 139.2 after intervention and mean diastolic blood pressure had decreased from 91.2 before intervention to 89.2 after intervention.

Burke et al (2007) observed the benefits of the lifestyle modification program provided to hypertensive patients and found that $89 \%$ of the studied subjects reported healthy behaviors as decrease dietary fat, increased physical activity, decrease weight, and increase fish and vegetable in diet.

The DASH diet lowered systolic blood pressure by an average of about $6 \mathrm{~mm} \mathrm{Hg}$ and diastolic pressure by about 3 $\mathrm{mm} \mathrm{Hg}$. The diet that was merely higher in fruits and vegetables also lowered blood pressure, but by a lesser amount: about $3 \mathrm{~mm} \mathrm{Hg}$ systolic and $2 \mathrm{mmHg}$ diastolic (Appel et al., 1997).

On the contrary the study of Seung-Hye, (2014) there was no significant change in blood pressure in both experimental and control groups. 
In this study there was no significant change in blood pressure in the control group (G2) (figure 2) because there was no change in knowledge nor in attitude of the patients. It was consistent with Habib et al., 2016.

\section{Conclusion and Recommendations}

We conclude from the results of the present study that the educational program for hypertensive patients about DASH diet proved its success in improving patient's knowledge, attitude and reduction of blood pressure level. So we recommend the following:

- Develop health education campaigns in mass media about importance of DASH diet and other life style modification in management of hypertension.

- Continuous training of physicians and providing them with recent guidelines to improve their knowledge about hypertension management.

\section{References}

[1] Al-Wehedy A., Abd Elhameed S., and Abd El-Hameed D., (2014): Effect of Lifestyle Intervention Program on Controlling Hypertension among Older Adults. Journal of Education and Practice ISSN 2222-1735 (Paper) ISSN 2222288X Vol. 5, No. 5.

[2] Appel L., Moore T., Obarzanek E., Vollmer W., Svetkey L. et al. (1997): A clinical trial of the effects of dietary patterns on blood pressure. DASH Collaborative ResearchGroup. N Engl J Med; 336: 1117-1124.

[3] Arafa N. and Ez-Elarab H., (2011): "Epidemiology of prehypertension and hypertension among Egyptian Adults." Egyptian Journal of Community Medicine 29, no.1:0-0. http://www.eacm.org.eg/PDF/2011/January_2011/january_11_ 01.pdf

[4] Burke V., Beilin L., Cutt H., Mansour J., Williams A. et al., (2007): A lifestyle program for treated hypertensives improved health-related behaviors and cardiovascular risk factors, a randomized controlled trial. Journal of Clinical Epidemiology; 60:133-41.

[5] Chen S., Maruthur N., and Appel L., (2010): The Effect of Dietary Patterns on Estimated Coronary Heart Disease Risk: Results From the Dietary Approaches to Stop Hypertension (DASH) Trial. Circulation: Cardiovascular Quality and Outcomes; DOI: 10.1161/CIRCOUTCOMES.109.930685

[6] Clark C., Taylor R., Shore A. and Campbell J., (2012): Association of a difference in systolic blood pressure between arms with vascular disease and mortality: a systematic review and meta-analysis. Lancet; 379:905-14. BMJ 344:e1327

[7] El-Gilany A, El-Wehady A and El-Wasify M., (2012): Updating and validation of the socioeconomic status scale for health research in Egypt. EMHJ;18:9.

[8] Ford C., Kim M. and Dancy B., (2009): Perceptions of hypertension and contributing personal and environmental factors among rural Southern African American women. Ethn Dis; 19:407-13.

[9] Habib D., Almoughazy M., Hassan A., and Orabi E., (2016):-
Effect of an educational intervention about Dietary Approach To Stop Hypertension (DASH) on Changing knowledge and Attitude of hypertensive Patients Attending Dierb negm hospital. Master thesis in Community Medicine Zagazig University

[10] Harrison A (2014): "Web-based Nutrition Education in Georgia Senior Centers: Pilot Test of a Dietary Approaches to Stopping Hypertension (DASH) Diet Website Prototype." Thesis, Georgia State University. http://scholarworks.gsu.edu/nutrition_theses/54

[11] Heller M., (2016): What Is the DASH Diet? Available at http://dashdiet.org/what_is_the_dash_diet.asp

[12] Jeng-liang T., (2011): Effect of a DASH nutrition education intervention on nutritional knowledge, dietary practice, self efficacy and blood pressure in hypertensive individual. Master's thesis school of nutrition and health science Taipei medical university.

[13] Kamran A., Shekarchi A., Sharifirad G., Sharifian E., and Shekarchi L., (2015): Evaluating Dietary Perceptions Education Program among Rural Hypertensive Patients on Commitment and Adherence to Healthy Diet. Jhhjournal. Volume: 1. Page: 7-12.

[14] Magadza C., Radloff S. and Srinivas S. (2009): The effect of an educational intervention on patients' knowledge about hypertension, beliefs about medicines, and adherence. Res Social Adm Pharm; 5:363-75.

[15] Mahajan H., Kazi Y., Sharma B., and Velhal G., (2012): Health Education: an Effective Intervention in Hypertensive Patients. International Journal of Recent Trends in Science And Technology, ISSN 2277-2812 E-ISSN 2249-8109, Volume 4, Issue 2, pp 77-82.

[16] (National Heart, Lung, and Blood Institute, 2015):Description of the DASH Eating Plan. Available on https://www.nhlbi.nih.gov/health/health-topics/topics/dash

[17] Ogedegbe G., Fernandez S., Fournier L., Silver S., Kong J., et al., (2013). The Counseling Older Adults to Control Hypertension $(\mathrm{COACH})$ trial: Design and methodology of a group-based lifestyle intervention for hypertensive minority older adults. Contemporary Clinical Trials; 35: 70-79.

[18] Pandeyt R., Agrawal A., Misra A., Vikram N., Misra P. et al., (2013): Population-based intervention for cardiovascular diseases related knowledge and behaviours in Asian Indian women. Indian Heart J. 2013; 65:40-7. [PMC free article] [PubMed]

[19] Park Y., Song M., Cho B., Lim J., Song W. et al., (2011). The effects of an integrated health education and exercise program in community-dwelling older adults with hypertension: A randomized controlled trial. Education and Counseling; 82(2): 133-37.

[20] Parmar P., Rathod G., Rathod S., Goyal R., Aggarwal S. et al., (2014): Study of knowledge, attitude and practice of general population of Gandhinagar towards hypertension. Int. J. Curr. Microbiol. App. Sci 3(8) 680-685.

[21] Rakumakoe M., (2011): "To determine the knowledge, attitudes and perceptions of hypertensive patients towards lifestyle modification in controlling hypertension" 04 OCTOBER partial fulfillment of MSc (Med) in the field of Sports Medicine, Student number: $9300793 \mathrm{H}$. 
[22] Sabouhi F., Babaee S., Naji H., and Zadeh A. (2011): Knowledge, awareness, attitudes and practice about hypertension in hypertensive patients referring to public health care centers in Khoor \& Biabanak. Iran J Nurs Midwifery Res. Winter; 16(1):34-40.

[23] Seung-Hye C. and Choi-Kwon S. (2014): The effects of the DASH diet education program with omega-3fatty acid supplementation on metabolic syndrome parameters in elderly women with abdominal obesity Nutrition Research and Practice. published in nrp, December 20. Available at: (http://creativecommons.org/licenses/by-nc/3.0/)

[24] Shaw R. and Bosworth H., (2012): Baseline medication adherence and blood pressure in a 24 month longitudinal hypertension study. J Clin Nurs;21:1401-1406. doi: 10.1111/j.1365-2702.2011.03859.x.

[25] Tadevosyan A., (2013): Knowledge, Attitude and Practice
(KAP) Survey of Hypertensive People. Master of Public Health Integrated Experience Project Professional Publication Framework School of Public Health, American University of Armenia Yerevan, Armenia.

[26] U.S. Department of Health and Human Services, (2006): "Your Guide To Lowering Your Blood Pressure With DASH" (PDF). April. Retrieved 2011-12-28.

[27] USDA (U.S. Department of Agriculture), (2010): "2010 Dietary Guidelines for Americans" (PDF) (Chapter 5 in 7 ed.). U.S. Government Printing Office. Retrieved December 15, 2014.

[28] WHO (World health organization), (2015): Q\& As on hypertension. Available at: http://www.who.int/features/qa/82/en/

[29] WHO (World health organization), (2015): Health education. Available at: http://www.who.int/topics/health_education/en/ 